\title{
The photographic observations on the components of Sirius
}

\author{
D.M.D. Jasinta and B. Hidayat
}

Bosscha Observatory, Lembang 40391, Bandung, Indonesia

Department of Astronomy, Institut Teknologi Bandung, Jl. Ganesha 10 Bandung 40132, Indonesia

Received October 28; accepted December 18, 1998

\begin{abstract}
A series of photographic observations on the components of Sirius was carried out in the years 19761986 at the Bosscha Observatory. We present the analysis and the results of the measurements ${ }^{1}$.
\end{abstract}

Key words: astrometry — binaries: visual — stars: individual: Sirius

\section{Introduction}

This is the continuation of the observations published by van Albada-van Dien (1977). We publish the results of photographic observations on the components of Sirius, made with the 60 -cm Zeiss double refractor at the Bosscha Observatory in the years 1976-1986.

\section{The observations}

A series of 54 plates photographed in 46 nights was collected with the $60-\mathrm{cm}$ Zeiss double refractor in the years 1976-1986. Plates with multiple exposures were taken using OG I or OG 530 filter and II G or $103 \mathrm{aG}$ emulsion. Trails were obtained to determine the direction of the zeropoint of the position angle.

A special "Sirius grating" designed by van Albada (1956) was placed in front of the objective lens. The distance between the wires is adjustable so as to make the distances of the first diffraction images from Sirius A equal to the distance A-B. By this arrangement the correction for contraction of the emulsion near the heavily overexposed zeroth order image of the primary can be derived.

The following grating constants $w / s$ were employed ( $w$ indicates the number of wires used in the grating, while $s$

Send offprint requests to: D.M.D. Jasinta,

e-mail: bosscha@ibm.net

1 Tables are also available in electronic form at the CDS via anonymous ftp 130.79.128.5 or http://cdsweb.ustrasbg.fr/Abstract.html denotes the standard spacing in $\mathrm{mm}$ ): 6/11 (plates 5602 7201), 5/11 (plates 7322-7401), 5/12 (plates 7781-8178), and $5 / 17$ for plates $8410-8449$. A hexagonal aperture was utilized to take plates 8039-8449.

\section{The measurements}

The plates were analysed using the Leitz UWM3 measuring machine. All plates were measured twice in positions differing by $180^{\circ}$, in order to eliminate remnants of magnitude error in setting on the large image.

Following the method used by van Albada-van Dien (1977), the relative positions of the components were obtained directly by measuring the secondary image and the zeroth order of the primary, instead of averaging the first or second diffraction images. This is to avoid asymmetric diffraction pattern due to centering error of the grating. The first $(b b)$ and second $(c c)$ order of Sirius A were also measured, to find the contraction of the emulsion, $C$, and to check on the asymmetry.

\section{The reductions}

The reductions were carried out on the basis of a plate scale of 19 .'169/mm. We found the coefficient of contraction of the emulsion of the plates in this series of observations is $C=1.0006 \pm 0.0073$ (mean of mean errors) for those taken with full diaphragm and $C=0.9982 \pm 0.0094$ for plates taken with the hexagonal aperture. Coefficients stated above indicate that there is an insignificant contraction effect.

In order to find the asymmetry on diffraction pattern, the averaged first order images of the primary were compared with the direct measurement of Sirius A. In each set of the two measurements, we found the same case with that discovered by van Albada-van Dien (1977). The difference is always in the same direction, namely, the averaged first diffraction image is always to the north of Sirius A (with a mean displacement of 0 "' $130 \pm 0$ ".'119 to the north). However, this systematic displacement does not hold for plates taken with hexagonal aperture. 
Table 1. Individual measurements

\begin{tabular}{|c|c|c|c|c|c|c|c|c|c|c|c|c|c|c|c|c|c|}
\hline PLATE & $\mathrm{EPOCH}$ & $\Delta \alpha \cos \delta$ & $\sigma$ & $\Delta \delta$ & $\sigma$ & $\theta$ & $\sigma$ & $\rho_{\mathrm{m}}$ & $\sigma$ & $C$ & $\sigma$ & $\rho_{\mathrm{c}}$ & $\sigma$ & $W_{\theta}$ & $\overline{W_{\rho}}$ & $n$ & OBS \\
\hline 5602 & 1976.962 & 8.931 & 47 & 6.281 & 40 & 54.88 & 22 & 10.919 & 45 & 1.0004 & 69 & 10.913 & 88 & 6 & 1 & 13 & SS \\
\hline 5603 & 1976.962 & 9.012 & 50 & 6.273 & 52 & 55.16 & 27 & 10.980 & 51 & 1.0015 & 51 & 10.964 & 75 & 4 & 2 & 15 & SS \\
\hline 5606 & 1976.978 & 8.991 & 62 & 6.559 & 111 & 53.89 & 50 & 11.129 & 82 & 0.9983 & 41 & 11.148 & 93 & 1 & 1 & 5 & DD \\
\hline 5607 & 1976.978 & 8.995 & 32 & 6.603 & 37 & 53.72 & 18 & 11.158 & 34 & 0.9980 & 33 & 11.180 & 50 & 8 & 4 & 10 & DD \\
\hline 5616 & 1977.003 & 9.114 & 51 & 6.325 & 73 & 55.24 & 34 & 11.094 & 59 & 0.9978 & 90 & 11.118 & 60 & 2 & 3 & 9 & SS \\
\hline 5619 & 1977.027 & 9.092 & 49 & 6.415 & 38 & 54.79 & 22 & 11.127 & 46 & 1.0089 & 84 & 11.029 & 103 & 6 & 1 & 12 & SS \\
\hline 5950 & 1977.852 & 8.591 & 88 & 6.668 & 78 & 52.18 & 43 & 10.875 & 84 & & & & & & & 3 & SS \\
\hline 5951 & 1977.855 & 8.689 & 42 & 6.612 & 56 & 52.73 & 27 & 10.919 & 48 & 0.9985 & 84 & 10.935 & 104 & 4 & 1 & 10 & SS \\
\hline 5965 & 1977.877 & 8.762 & 60 & 6.520 & 56 & 53.35 & 30 & 10.922 & 59 & & & & & & & 10 & SS \\
\hline 5966 & 1977.877 & 8.640 & 81 & 6.489 & 63 & 53.09 & 37 & 10.805 & 75 & 0.9959 & 50 & 10.850 & 93 & 2 & 1 & 7 & SS \\
\hline 5974 & 1977.937 & 8.651 & 58 & 6.395 & 87 & 53.53 & 42 & 10.758 & 70 & & & & & & & 5 & SS \\
\hline 5975 & 1977.937 & 8.643 & 53 & 6.507 & 73 & 53.03 & 35 & 10.819 & 61 & 1.0009 & 66 & 10.810 & 94 & 2 & 1 & 7 & SS \\
\hline 5978 & 1977.953 & 8.849 & 75 & 6.403 & 64 & 54.11 & 36 & 10.923 & 71 & 0.9993 & 45 & 10.930 & 86 & 2 & 1 & 5 & SS \\
\hline 6208 & 1978.819 & 8.143 & 69 & 6.737 & 40 & 50.40 & 29 & 10.569 & 59 & 0.9976 & 52 & 10.579 & 81 & 4 & 2 & 10 & SW \\
\hline 6209 & 1978.822 & 8.219 & 56 & 6.758 & 39 & 50.57 & 25 & 10.641 & 50 & 1.0024 & 56 & 10.615 & 78 & 5 & 2 & 6 & SW \\
\hline 6212 & 1978.830 & 8.104 & 31 & 6.990 & 45 & 49.22 & 21 & 10.702 & 38 & 1.0004 & 59 & 10.698 & 73 & 7 & 2 & 16 & MR \\
\hline 6216 & 1978.836 & 8.176 & 48 & 6.931 & 53 & 49.71 & 27 & 10.718 & 50 & & & & & & & 9 & MR \\
\hline 6224 & 1978.841 & 8.314 & 71 & 6.720 & 44 & 51.05 & 30 & 10.690 & 62 & 0.9993 & 64 & 10.697 & 92 & 3 & 1 & 11 & MR \\
\hline 6261 & 1979.079 & 8.236 & 62 & 7.028 & 43 & 49.52 & 27 & 10.827 & 55 & 1.0019 & 106 & 10.807 & 127 & 4 & 1 & 11 & MR \\
\hline 6711 & 1979.945 & 7.647 & 109 & 6.967 & 79 & 47.66 & 52 & 10.345 & 97 & & & & & & & 7 & AW \\
\hline 6712 & 1979.948 & 7.879 & 86 & 6.988 & 51 & 48.43 & 37 & 10.531 & 73 & & & & & & & 9 & MR \\
\hline 6713 & 1979.964 & 7.742 & 37 & 7.117 & 45 & 47.41 & 23 & 10.516 & 41 & 1.0033 & 64 & 10.482 & 78 & 6 & 2 & 12 & AW \\
\hline 6714 & 1979.967 & 7.721 & 53 & 7.019 & 33 & 47.73 & 24 & 10.435 & 45 & & & & & & & 16 & MR \\
\hline 6720 & 1980.025 & 7.726 & 77 & 6.851 & 78 & 48.44 & 43 & 10.326 & 77 & & & & & & & 9 & AW \\
\hline 6731 & 1980.068 & 7.680 & 65 & 7.139 & 70 & 47.09 & 37 & 10.485 & 67 & 1.0028 & 80 & 10.457 & 107 & 2 & 1 & 5 & AW \\
\hline 6732 & 1980.068 & 7.698 & 110 & 6.959 & 112 & 47.89 & 61 & 10.377 & 111 & & & & & & & 5 & AW \\
\hline 6743 & 1980.126 & 7.611 & 48 & 7.039 & 80 & 47.24 & 37 & 10.367 & 65 & 1.0057 & 63 & 10.309 & 91 & 2 & 1 & 11 & MR \\
\hline 6748 & 1980.128 & 7.669 & 145 & 6.919 & 77 & 47.94 & 63 & 10.329 & 119 & 1.0056 & 61 & 10.267 & 134 & 1 & 1 & 4 & AW \\
\hline 6765 & 1980.161 & 7.770 & 53 & 7.048 & 52 & 47.79 & 29 & 10.491 & 53 & 1.0020 & 87 & 10.470 & 107 & 4 & 1 & 8 & AW \\
\hline 7130 & 1980.762 & 7.427 & 33 & 7.124 & 49 & 46.19 & 23 & 10.291 & 41 & 1.0003 & 66 & 10.288 & 79 & 6 & 2 & 14 & AW \\
\hline 7152 & 1980.811 & 7.273 & 88 & 6.795 & 54 & 46.95 & 42 & 9.907 & 74 & 1.0057 & 113 & 9.851 & 133 & 2 & 1 & 7 & MR \\
\hline 7183 & 1981.014 & 7.372 & 90 & 7.216 & 100 & 45.61 & 53 & 10.316 & 95 & 1.0064 & 50 & 10.250 & 107 & 1 & 1 & 3 & AW \\
\hline 7187 & 1981.055 & 7.247 & 43 & 7.137 & 43 & 45.44 & 24 & 10.171 & 43 & 0.9980 & 96 & 10.192 & 107 & 6 & 1 & 11 & MR \\
\hline 7195 & 1981.110 & 7.067 & 73 & 7.041 & 46 & 45.11 & 35 & 9.976 & 61 & 1.0047 & 91 & 9.929 & 109 & 3 & 1 & 10 & MR \\
\hline 7322 & 1981.729 & 6.806 & 42 & 7.071 & 33 & 43.91 & 22 & 9.814 & 38 & & & & & & & 11 & AW \\
\hline 7325 & 1981.742 & 6.835 & 45 & 7.127 & 52 & 43.80 & 28 & 9.875 & 49 & 1.0092 & 55 & 9.786 & 72 & 4 & 2 & 11 & $\mathrm{D}$ \\
\hline 7332 & 1981.756 & 6.708 & 37 & 7.228 & 32 & 42.86 & 20 & 9.861 & 34 & 1.0014 & 83 & 9.847 & 88 & 9 & 1 & 12 & AW \\
\hline 7393 & 1981.847 & 6.798 & 51 & 7.082 & 28 & 43.83 & 24 & 9.817 & 41 & 1.0004 & 83 & 9.813 & 91 & 6 & 1 & 11 & MR \\
\hline 7401 & 1981.956 & 6.680 & 54 & 7.170 & 39 & 42.98 & 28 & 9.800 & 47 & 1.0004 & 69 & 9.796 & 82 & 4 & 2 & 8 & $\mathrm{EP}$ \\
\hline 7781 & 1983.923 & 5.617 & 45 & 6.824 & 105 & 39.46 & 49 & 8.838 & 86 & & & & & & & 4 & $\mathrm{EP}$ \\
\hline 7791 & 1983.932 & 5.649 & 139 & 7.021 & 45 & 38.82 & 71 & 9.011 & 94 & & & & & & & 6 & $\mathrm{EP}$ \\
\hline 7912 & 1984.863 & 4.829 & 34 & 6.851 & 34 & 35.18 & 23 & 8.382 & 34 & 1.0006 & 88 & 8.377 & 81 & 9 & 2 & 19 & EP \\
\hline 7914 & 1984.880 & 4.803 & 45 & 6.871 & 44 & 34.95 & 31 & 8.383 & 44 & 0.9937 & 79 & 8.437 & 80 & 5 & 2 & 7 & $\mathrm{EP}$ \\
\hline 7920 & 1984.888 & 4.892 & 35 & 6.870 & 25 & 35.45 & 22 & 8.434 & 29 & 0.9933 & 117 & 8.491 & 105 & 9 & 1 & 16 & EP \\
\hline 7924 & 1985.020 & 4.776 & 45 & 6.888 & 28 & 34.74 & 28 & 8.382 & 34 & 0.9819 & 92 & 8.537 & 87 & 6 & 1 & 3 & $\mathrm{EP}$ \\
\hline 7935 & 1985.071 & 4.738 & 59 & 6.721 & 55 & 35.18 & 40 & 8.223 & 56 & 1.0041 & 99 & 8.190 & 98 & 3 & 1 & 15 & $\mathrm{EP}$ \\
\hline 8039 & 1986.085 & 4.131 & 158 & 6.441 & 76 & 32.67 & 104 & 7.652 & 107 & 0.9975 & 75 & 7.675 & 122 & 1 & 1 & 2 & $\mathrm{EP}$ \\
\hline 8135 & 1986.825 & 3.228 & 73 & 6.307 & 120 & 27.10 & 69 & 7.085 & 112 & 1.0033 & 97 & 7.062 & 131 & 1 & 1 & 3 & $\mathrm{EP}$ \\
\hline 8137 & 1986.827 & 3.259 & 55 & 6.329 & 39 & 27.25 & 42 & 7.119 & 43 & 0.9966 & 89 & 7.143 & 77 & 4 & 2 & 9 & $\mathrm{EP}$ \\
\hline 8138 & 1986.827 & 3.174 & 47 & 6.354 & 40 & 26.54 & 37 & 7.103 & 41 & 0.9906 & 106 & 7.171 & 87 & 5 & 1 & 9 & $\mathrm{EP}$ \\
\hline 8147 & 1986.921 & 3.189 & 57 & 6.422 & 98 & 26.41 & 55 & 7.100 & 92 & 0.9999 & 132 & 7.101 & 131 & 2 & 1 & 5 & $\mathrm{EP}$ \\
\hline 8148 & 1986.921 & 3.057 & 55 & 6.402 & 113 & 25.53 & 56 & 7.095 & 105 & 1.0027 & 42 & 7.074 & 109 & 2 & 1 & 2 & $\mathrm{EP}$ \\
\hline 8154 & 1986.929 & 3.114 & 51 & 6.275 & 82 & 26.39 & 48 & 7.005 & 77 & 0.9983 & 106 & 7.017 & 107 & 3 & 1 & 6 & $\mathrm{EP}$ \\
\hline 8155 & 1986.929 & 3.157 & 37 & 6.377 & 49 & 26.34 & 32 & 7.116 & 47 & 0.9965 & 107 & 7.141 & 90 & 6 & 1 & 7 & $\mathrm{EP}$ \\
\hline
\end{tabular}


For these plates, the difference is always to the north for one position (with trail below the images), and to the south for the other position (trail above the images after a $180^{\circ}$ rotation). The mean value to the north is $0^{\prime \prime} .111 \pm$ 0 .'062, and the mean value to the south is $0 . \prime 082 \pm 0$. . 075 . Contrary to what was detected by van Albada-van Dien (1977), we do not find the tendency to east or west displacements, nor the same direction of displacements in series of plates taken in succession is found.

The observations were weighted with the inverse square of the mean error, without another addition for plate or night errors. Weight 1 was attributed to a mean error of 0 .' 1 in $\rho_{\mathrm{c}}$ and of $0.1 / \rho_{\mathrm{c}}^{\prime \prime}$ radians in $\theta$. For fair quality plates, we often could not measure the first and/or the second diffraction images. For these kind of plates, the contraction of emulsion was not defined, and therefore weights could not be assigned.

The results of the reductions appear in Table 1, which consists of:

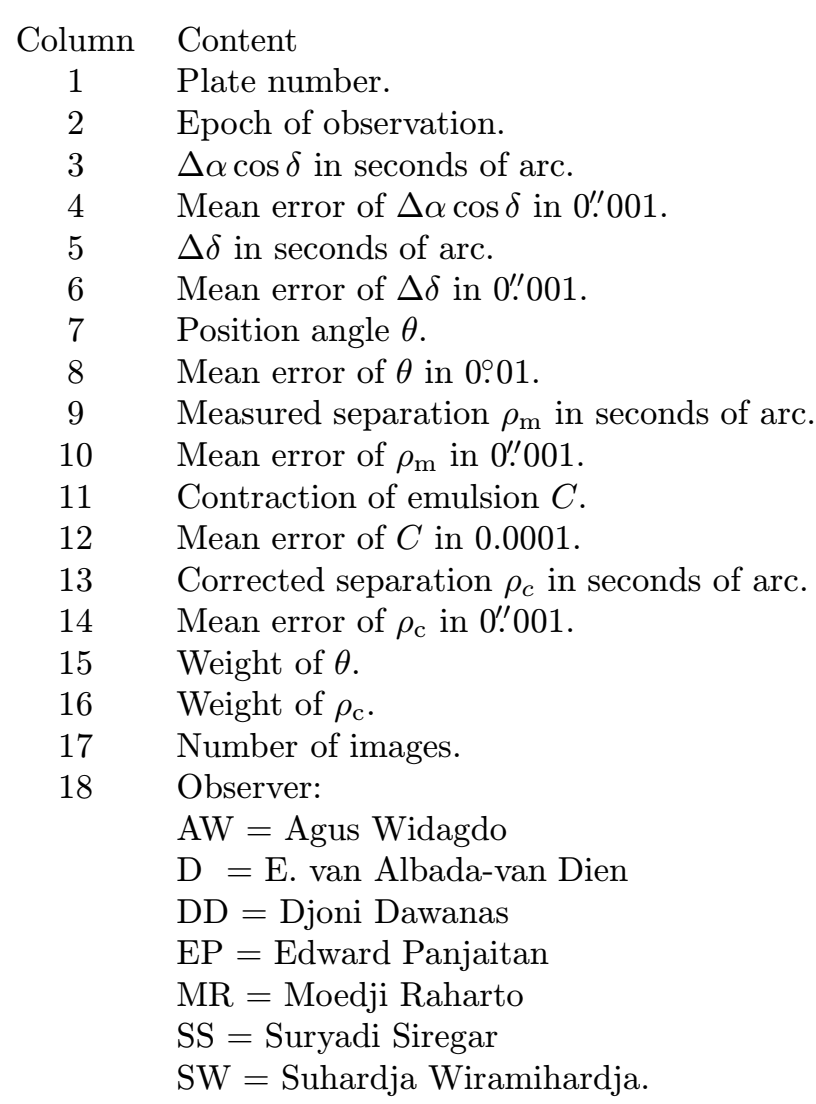

Table 2. Weighted yearly means

\begin{tabular}{ccrcrrr}
\hline Epoch & $\theta$ & $W_{\theta}$ & Epoch & $\rho$ & $W_{\rho}$ & $N$ \\
\hline 1976.985 & 54.54 & 27 & 1976.984 & 11.089 & 12 & 6 \\
1977.898 & 53.15 & 10 & 1977.909 & 10.880 & 5 & 4 \\
1978.873 & 50.02 & 23 & 1978.849 & 10.660 & 8 & 5 \\
1979.964 & 47.41 & 6 & 1979.964 & 10.482 & 2 & 1 \\
1980.126 & 47.49 & 9 & 1980.120 & 10.379 & 4 & 4 \\
1980.933 & 45.83 & 18 & 1980.942 & 10.132 & 6 & 5 \\
1981.814 & 43.31 & 23 & 1981.821 & 9.807 & 6 & 4 \\
1983.926 & 39.26 & & 1983.927 & 8.914 & & 2 \\
1984.921 & 35.15 & 32 & 1984.942 & 8.412 & 7 & 5 \\
1986.085 & 32.67 & 1 & 1986.085 & 7.675 & 1 & 1 \\
1986.885 & 26.51 & 23 & 1986.876 & 7.114 & 8 & 7 \\
\hline
\end{tabular}

Table 2 yields the yearly means. Only plates with weights were included, except for those taken in 1983 which have no weights.

$\begin{array}{cl}\text { Column } & \text { Content } \\ 1 & \text { Mean epoch for } \theta \\ 2 & \text { Mean value of } \theta \\ 3 & \text { Weight of mean value of } \theta \\ 4 & \text { Mean epoch for } \rho_{\mathrm{c}} . \\ 5 & \text { Mean value of } \rho_{\mathrm{c}} . \\ 6 & \text { Weight of mean value of } \rho_{\mathrm{c}} . \\ 7 & \text { Number of plates. }\end{array}$

We are still investigating the systematic errors which might come from the geometric effect. In the future, we propose that further photographic measurements are carried out in order to study the effect.

Acknowledgements. The authors would like to express their sincerest gratitude to the Director of Leids Kerkhoven-Bosscha Fonds for the generous support on the research of double stars at the Bosscha Observatory. They also acknowledge Dr. E. van Albada-van Dien and Dr. Taufiq Hidayat for the suggestions and fruitful discussions, and Edy Susilo for measuring the plates.

\section{References}

van Albada G.B., 1956, Contr. Bosscha Obs., No. 3 van Albada-van Dien E., 1977, A\&AS 29, 305 